\title{
Stressors: A Challenge for the Faculty Members of the Higher Educational Institutions
}

\author{
Ekta Sharma \\ Asst. Professor, Amrut Mody School of Management, \\ Ahmedabad University, India \\ E-mail: ektas55@gmail.com
}

Received: Nov. 10, $2014 \quad$ Accepted: Nov. 27, $2014 \quad$ Published: Nov. 30, 2014

doi:10.5296/csbm.v1i2.6711 URL: http://dx.doi.org/10.5296/csbm.v1i2.6711

\begin{abstract}
There is a lot of research in the area of stress. The studies consider that the stressors vary according to the environment. With the changing environment, the higher education institutions also need to develop their competitive advantage and hence make it challenging for the stakeholders, especially the faculty members to contribute towards the competitive advantage. This leads to stress amongst the faculty members. The current paper is an exploratory research to investigate the role stressors of the faculty members of various universities from different countries. The research is based on Organizational Role Stress Instrument of Udai Pareek (2002).
\end{abstract}

Keywords: Organizational Role Stress, VUCA, Faculty members, Stressors 


\section{Introduction}

Traditionally the faculty's job has not been considered stressful and hence there is a sparse research on this topic. But in the VUCA world the situations has changed and to corroborate that, the forbes magazine's survey concludes that the University Professors' job is not least stressful Job. (Forbes, 2013). Much can be attributed to the changing work demands, change in the policy and social status of higher Education.

The research demands are increasing and the faculty works in the environment of "publish or perish". Besides that, the faculty members have to work with an increasing number of students, who are also more demanding. The industry demands are changing and hence the faculty have to review the curriculum to adapt to these changes. They are also assigned administrative duties and all this together make their working day longer and stressful. The increasing expectation from the role as a faculty demands them to work mentally or physically for around twenty to twenty three hours and to aggravate that is the expectation of family and society. The sample size of current study is the faculty members of the higher education institutions.

\section{Literature Review}

The research in the area of stress is wide and varied. But not has been done to analyze the stress levels of the faculty members, teaching in the higher educational institutions. Besides that Occupational stress has been the focus of research and the role stress is not studied.

The literature shows that there are significant differences in the stress level when studied in reference to tenure, rank, age, gender and marital status. (Gmelch, Willse, \& Lourich, 1986; Smith \& Sanders, 1989; Slišković \& Maslić, 2011). The other studies have proved the difference in the stress level on the basis of designation and work experience (Madhu, 1990; Chaudhary, 2013).

The stress levels of the faculty members also differ as per their designation, which seems to be a significant point to study role stress, as the change in designation leads to change in role. Slišković and Maslić (2011) show that the Full professors, reported lower exposure to stress at work than associate professors, assistant professors, and assistants.

The other studies have proved that the stress levels of females are more than males (Kausar \& Bashir, 2004; Jreige, 2011; Ravichandran \& Rajendran, 2007; Manthei, 1988; Spielberger \& Reheiser, 1994; Repetti \& Wood, 1997). But this is contradicted by the study of Kavitha (2012) and Areekkuzhiyil (2011) whose research proves that males have higher stress levels than the females. This is another point which makes it significant to study role stress as the difference in the results of the above researches can be attributed to the difference in their role.

Arnold and Feldman (1986) explored number of potential sources of job stress experienced by different persons and concluded that the role under load, role overload, role conflict, role ambiguity, and job characteristics are the major sources of the job stress.

Cooper, Dewe, \& O'Driscoll (2001) have proved that role conflict is one of the significant stressor. 


\section{Macrothink}

The faculty members are under stress (Gmelch, 1993; Dinham \& Scott, 1998; Kyriacou, 2001; Kavitha, 2012; Mishra et al., 1997; Spector, 1997; Mohsin, 2004).

Kavitha (2012) concluded that the role overload and role conflict are the significant stressors for the faculty members, which are also noticed by Mishra et al. (1997), Spector (1997) and Mohsin (2004). The important discriminate role stress factors among the stressful and stress-free faculty members are role overload, and self role distance. In fact, the faculty members are stressed even because of the multiple task that they are required to handle, besides teaching. Not every faculty member have flair for administration but all the faculty members are assigned some or the other administrative responsibilities, which they find difficult to handle and also find themselves distant from this role.

The literature has a huge gap, in the study of role stress amongst the faculty members of higher educational Institutions and hence, the article has been an effort to bridge this gap. The study is conducted to find out the stress levels of faculty members from different institutions of different countries.

\section{Research Methodology}

The data is collected through Organizational Role stress instrument (Pareek, 2004). The sample size of the study is 108 , out of which 40 are females and 68 are males. The sample consists of faculty members from the institutions of various countries like India, UAE, some African countries, US etc. The Independent Sample t-test has been conducted to analyze the data.

\subsection{Organisational Role Stress (ORS)}

Organizational role is a position assigned to the individual, in the organization, which is defined by the expectations of the stakeholders for that role. The role occupant performs certain functions in the organization in response to his/her role expectation (Pareek, 2004). The concept of organizational role has in-built potential for stress. Stress from occupation of an organization role and performing therein, is known as Organizational Role Stress (ORS). Framework of ORS developed by Pareek (1983) defines ten components of a role stress. These are: i) Inter-Role Distance; ii) Role Stagnation; iii) Role Expectation Conflict; iv) Role Erosion; v) Role Over load; vi) Role Isolation; vii) Personal Inadequacy; viii) Self Role Distance; ix) Role ambiguity; and x) Resource inadequacy.

Inter-role Distance (IRD): When there is conflict between the organizational role and other roles, e.g., an executive not being able to divide his time between work demands and family demands.

Role Stagnation (RS): when there are few opportunities for learning and growth in organization.

Role Expectations Conflict (REC): When there are conflicting demands made on the role by different people in the organization. 
Role Erosion (RE): When an individual feels that some important functions which are related to his work are given to someone else to carryout he/she feels that the job which he is doing is not challenging. The stress indicators found to be related to role erosion are a feeling of worthlessness, low self-esteem, mood swing, low motivation to work, etc.

Role Overload (RO): When there is a feeling that too much is expected from the role than what the occupant can cope with.

Role Isolation (RI): When there is a lack of appropriate linkage of one's role with the others' role in the organization

Personal Inadequacy (PI): When there is a lack of knowledge, skills or adequate preparation to be effective in a particular role.

Self-Role Distance (SRD): When there is conflict between one's values and self-concepts with the requirements of the organizational role.

Role Ambiguity (RA): When an individual does not have a clear picture of work objectives, co-workers' expectations and the scope and responsibilities of his/her job. The stress indicators found to relate to role ambiguity and depressed mood, lowered self-esteem, low motivation to work and intention to leave the job.

Resource Inadequacy (RI): When there is non-availability of resources needed for effective role performance.

\subsection{Hypothesis}

The literature review provides evidence that the stress levels of females are more than males. (Kausar, Fatima, \& Bashir, 2004; Jreige, 2011; Ravichandran \& Rajendran, 2007; Manthei, 1988; Spielberger \& Reheiser, 1994; Repetti \& Wood, 1997).

1) The stress levels of the faculty members are not significantly different on basis of gender.

2) The stress levels of faculty members of Government Institutions is less than the faculty members of private owned Institutions.

There is difference in the stress level on the basis of designation and work experience (Madhu, 1990; Chaudhary, 2013).

The stress levels are not significantly related to the work experience of the faculty members.

\subsection{Hypothesis Testing}

1) The stress levels of the faculty members are not significantly different on basis of gender.

Table 2 shows that there is no significant difference in the stress level of the faculty members on basis of gender. As the significance value for the variable stress is .119.

Hence, the hypothesis is accepted.

2) The stress levels of faculty members of Government Institutions is less than the faculty members of private owned Institutions. 


\section{Macrothink}

The stress levels of the faculty members in the government institution have higher stress levels (Mean=125) than the stress levels of their counterparts in the private institutions (Mean=58.5) (Table 3). Even Table 4 shows the value of "p" is 0.

Hence, the hypothesis is rejected.

3) The stress levels are not significantly related to the work experience of the faculty members.

The stress levels of the faculty members with experience less than 5 years (Mean=51.5) is less than those with the experience more than 5 years (Mean=70) (Table 5). The table 6 also shows that the ' $p$ ' value is 0.008 . This shows that the hypothesis is accepted.

\section{Findings}

The research shows that the role stress is prevalent amongst the faculty members of the higher Educational institutions. Although the stress levels does not have significant difference, on the basis of gender, but, there is a difference. The stress level of male faculty members (61) is less than the female faculty members (71). (Table 1). The table 2 shows that except IRD, RI and RIn, all other stress variables differ as per the gender. Table 3 and 4 shows that the stress level of the faculty members in government owned institution is higher than the private owned institution. The faculty with more than 5 years of work experience, exhibits higher stress than their colleagues with less experience in job, which can be attributed to increasing expectation from the faculty members, who have higher work experience. It is expected of them to take up significant administrative role and also to devote time towards research.

Table 1. Descriptive statistics of stress on basis of gender

\begin{tabular}{|ll|r|r|r|c|}
\multicolumn{7}{c}{ Group Statistics } \\
\hline & Gender(0=M,1=F) & $\mathrm{N}$ & Mean & Std. Deviation & $\begin{array}{c}\text { Std. Error } \\
\text { Mean }\end{array}$ \\
\hline IRD & 0 & 60 & 8.40 & 6.137 & .792 \\
& 1 & 48 & 8.00 & 4.105 & .593 \\
\hline RS & 0 & 60 & 6.40 & 4.798 & .619 \\
& 1 & 48 & 9.00 & 3.713 & .536 \\
\hline REC & 0 & 60 & 5.60 & 1.870 & .241 \\
& 1 & 48 & 7.00 & 4.105 & .593 \\
\hline RE & 0 & 60 & 4.00 & 3.827 & .494 \\
& 1 & 48 & 6.00 & 4.347 & .627 \\
\hline RO & 0 & 60 & 6.80 & 5.158 & .666 \\
& 1 & 48 & 8.50 & 3.679 & .531 \\
\hline RI & 0 & 60 & 5.40 & 5.046 & .651 \\
& 1 & 48 & 6.75 & 3.145 & .454 \\
\hline PI & 0 & 60 & 2.60 & 2.264 & .292 \\
& 1 & 48 & 4.75 & 3.739 & .540 \\
\hline SRD & 0 & 60 & 9.60 & 3.227 & .417 \\
& 1 & 48 & 6.25 & 1.495 & .216 \\
\hline RA & 0 & 60 & 5.40 & 3.411 & .440 \\
& 1 & 48 & 7.00 & 4.405 & .636 \\
\hline Rin & 0 & 60 & 7.20 & 5.683 & .734 \\
& 1 & 48 & 8.25 & 2.709 & .391 \\
\hline Stress & 0 & 60 & 61.40 & 36.708 & 4.739 \\
& 1 & 48 & 71.50 & 28.210 & 4.072 \\
\hline
\end{tabular}


Table 2. Independent sample t test of stress on basis of gender

\begin{tabular}{|c|c|c|c|c|c|c|c|c|c|c|}
\hline \multicolumn{11}{|c|}{ Independent Samples Test } \\
\hline & & \multicolumn{2}{|c|}{$\begin{array}{l}\text { Levene's Test for } \\
\text { Equality of Variances }\end{array}$} & \multicolumn{7}{|c|}{ t-test for Equality of Means } \\
\hline & & \multirow[b]{2}{*}{$\mathrm{F}$} & \multirow[b]{2}{*}{ Sig. } & \multirow[b]{2}{*}{$\mathrm{t}$} & \multirow[b]{2}{*}{ df } & \multirow[b]{2}{*}{ Sig. (2-tailed) } & \multirow{2}{*}{$\begin{array}{c}\text { Mean } \\
\text { Difference }\end{array}$} & \multirow{2}{*}{$\begin{array}{l}\text { Std. Error } \\
\text { Difference }\end{array}$} & \multicolumn{2}{|c|}{$\begin{array}{l}95 \% \text { Confidence } \\
\text { Interval of the } \\
\text { Difference }\end{array}$} \\
\hline & & & & & & & & & Lower & Upper \\
\hline IRD & $\begin{array}{l}\text { Equal variances } \\
\text { assumed }\end{array}$ & \multirow[t]{2}{*}{2.678} & \multirow[t]{2}{*}{.105} & \multirow{2}{*}{$\begin{array}{l}.387 \\
.404\end{array}$} & \multirow{2}{*}{$\begin{array}{r}106 \\
103.002\end{array}$} & \multirow{2}{*}{$\begin{array}{l}.699 \\
.687\end{array}$} & \multirow{2}{*}{$\begin{array}{l}.400 \\
.400\end{array}$} & \multirow{2}{*}{$\begin{array}{r}1.033 \\
.989\end{array}$} & \multirow{2}{*}{$\begin{array}{l}-1.647 \\
-1.562\end{array}$} & \multirow{2}{*}{$\begin{array}{l}2.447 \\
2.362\end{array}$} \\
\hline & $\begin{array}{l}\text { Equal variances } \\
\text { not assumed }\end{array}$ & & & & & & & & & \\
\hline \multirow[t]{2}{*}{ RS } & $\begin{array}{l}\text { Equal variances } \\
\text { assumed }\end{array}$ & \multirow[t]{2}{*}{6.364} & \multirow[t]{2}{*}{.013} & \multirow{2}{*}{$\begin{array}{l}-3.086 \\
-3.174\end{array}$} & \multirow{2}{*}{$\begin{array}{r}106 \\
105.900\end{array}$} & \multirow{2}{*}{$\begin{array}{l}.003 \\
.002\end{array}$} & \multirow{2}{*}{$\begin{array}{l}-2.600 \\
-2.600\end{array}$} & \multirow{2}{*}{$\begin{array}{l}.843 \\
.819\end{array}$} & \multirow{2}{*}{$\begin{array}{l}-4.270 \\
-4.224\end{array}$} & \multirow{2}{*}{$\begin{array}{l}-.930 \\
-.976\end{array}$} \\
\hline & $\begin{array}{l}\text { Equal variances } \\
\text { not assumed }\end{array}$ & & & & & & & & & \\
\hline \multirow[t]{2}{*}{ REC } & $\begin{array}{l}\text { Equal variances } \\
\text { assumed }\end{array}$ & 40.775 & .000 & -2.356 & 106 & .020 & -1.400 & .594 & -2.578 & -.222 \\
\hline & $\begin{array}{l}\text { Equal variances } \\
\text { not assumed }\end{array}$ & & & -2.188 & 62.534 & .032 & -1.400 & .640 & -2.679 & -.121 \\
\hline $\mathrm{RE}$ & $\begin{array}{l}\text { Equal variances } \\
\text { assumed }\end{array}$ & 4.895 & .029 & -2.540 & 106 & .013 & -2.000 & .787 & -3.561 & -.439 \\
\hline & $\begin{array}{l}\text { Equal variances } \\
\text { not assumed }\end{array}$ & & & -2.505 & 94.433 & .014 & -2.000 & .799 & -3.585 & -.415 \\
\hline $\mathrm{RO}$ & $\begin{array}{l}\text { Equal variances } \\
\text { assumed }\end{array}$ & 8.584 & .004 & -1.925 & 106 & .057 & -1.700 & .883 & -3.451 & .051 \\
\hline & $\begin{array}{l}\text { Equal variances } \\
\text { not assumed }\end{array}$ & & & -1.996 & 104.730 & .049 & -1.700 & .852 & -3.389 & -.011 \\
\hline RI & $\begin{array}{l}\text { Equal variances } \\
\text { assumed }\end{array}$ & 8.256 & .005 & -1.618 & 106 & .109 & -1.350 & .834 & -3.004 & .304 \\
\hline & $\begin{array}{l}\text { Equal variances } \\
\text { not assumed }\end{array}$ & & & -1.700 & 100.475 & .092 & -1.350 & .794 & -2.925 & .225 \\
\hline $\mathrm{PI}$ & $\begin{array}{l}\text { Equal variances } \\
\text { assumed }\end{array}$ & 20.068 & .000 & -3.690 & 106 & .000 & -2.150 & .583 & -3.305 & -.995 \\
\hline & $\begin{array}{l}\text { Equal variances } \\
\text { not assumed }\end{array}$ & & & -3.503 & 73.574 & .001 & -2.150 & .614 & -3.373 & -.927 \\
\hline SRD & $\begin{array}{l}\text { Equal variances } \\
\text { assumed }\end{array}$ & 10.067 & .002 & 6.640 & 106 & .000 & 3.350 & .504 & 2.350 & 4.350 \\
\hline & $\begin{array}{l}\text { Equal variances } \\
\text { not assumed }\end{array}$ & & & 7.141 & 87.030 & .000 & 3.350 & .469 & 2.418 & 4.282 \\
\hline RA & $\begin{array}{l}\text { Equal variances } \\
\text { assumed }\end{array}$ & 2.119 & .148 & -2.128 & 106 & .036 & -1.600 & .752 & -3.091 & -.109 \\
\hline & $\begin{array}{l}\text { Equal variances } \\
\text { not assumed }\end{array}$ & & & -2.069 & 86.962 & .042 & -1.600 & .773 & -3.137 & -.063 \\
\hline Rin & $\begin{array}{l}\text { Equal variances } \\
\text { assumed }\end{array}$ & 45.507 & .000 & -1.177 & 106 & .242 & -1.050 & .892 & -2.819 & .719 \\
\hline & $\begin{array}{l}\text { Equal variances } \\
\text { not assumed }\end{array}$ & & & -1.263 & 88.335 & .210 & -1.050 & .831 & -2.702 & .602 \\
\hline Stress & $\begin{array}{l}\text { Equal variances } \\
\text { assumed }\end{array}$ & 2.190 & .142 & -1.571 & 106 & .119 & -10.100 & 6.431 & -22.850 & 2.650 \\
\hline & $\begin{array}{l}\text { Equal variances } \\
\text { not assumed }\end{array}$ & & & -1.617 & 105.850 & .109 & -10.100 & 6.248 & -22.488 & 2.288 \\
\hline
\end{tabular}




\section{Macrothink}

ISSN 2333-3324

2014, Vol. 1, No. 2

Table 3. Descriptive statistics of stress on basis of ownership of the institute (government or private)

Group Statistics

\begin{tabular}{|ll|r|r|r|r|}
\hline & Private/Govt(Private=1) & $\mathrm{N}$ & Mean & Std. Deviation & $\begin{array}{c}\text { Std. Error } \\
\text { Mean }\end{array}$ \\
\hline IRD & 0 & 12 & 19.00 & .000 & .000 \\
& 1 & 96 & 6.88 & 3.907 & .399 \\
\hline RS & 0 & 12 & 12.00 & .000 & .000 \\
& 1 & 96 & 7.00 & 4.496 & .459 \\
\hline REC & 0 & 12 & 9.00 & .000 & .000 \\
& 1 & 96 & 5.88 & 3.156 & .322 \\
\hline RE & 0 & 12 & 11.00 & .000 & .000 \\
& 1 & 96 & 4.13 & 3.776 & .385 \\
\hline RO & 0 & 12 & 14.00 & .000 & .000 \\
& 1 & 96 & 6.75 & 4.258 & .435 \\
\hline RI & 0 & 12 & 14.00 & .000 & .000 \\
& 1 & 96 & 5.00 & 3.482 & .355 \\
\hline PI & 0 & 12 & 5.00 & .000 & .000 \\
& 1 & 96 & 3.38 & 3.332 & .340 \\
\hline SRD & 0 & 12 & 15.00 & .000 & .000 \\
& 1 & 96 & 7.25 & 1.995 & .204 \\
\hline RA & 0 & 12 & 9.00 & .000 & .000 \\
& 1 & 96 & 5.75 & 4.044 & .413 \\
\hline Rin & 0 & 12 & 17.00 & .000 & .000 \\
& 1 & 96 & 6.50 & 3.409 & .348 \\
\hline StresS & 0 & 12 & 125.00 & .000 & .000 \\
& 1 & 96 & 58.50 & 27.617 & 2.819 \\
\hline
\end{tabular}


Table 4. Independent sample t-test of stress on basis of ownership of the institute (government or private)

\begin{tabular}{|c|c|c|c|c|c|c|c|c|c|c|}
\hline & & \multicolumn{2}{|c|}{$\begin{array}{c}\text { Levene's Test for } \\
\text { Equality of Variances }\end{array}$} & \multicolumn{7}{|c|}{ t-test for Equality of Means } \\
\hline & & \multirow[b]{2}{*}{$\mathrm{F}$} & \multirow[b]{2}{*}{ Sig. } & \multirow[b]{2}{*}{$t$} & \multirow[b]{2}{*}{ df } & \multirow[b]{2}{*}{ Sig. (2-tailed) } & \multirow{2}{*}{$\begin{array}{c}\text { Mean } \\
\text { Difference }\end{array}$} & \multirow{2}{*}{$\begin{array}{l}\text { Std. Error } \\
\text { Difference }\end{array}$} & \multicolumn{2}{|c|}{$\begin{array}{l}\text { 95\% Confidence } \\
\text { Interval of the } \\
\text { Difference }\end{array}$} \\
\hline & & & & & & & & & Lower & Upper \\
\hline IRD & $\begin{array}{l}\text { Equal variances } \\
\text { assumed } \\
\text { Equal variances } \\
\text { not assumed }\end{array}$ & 78.612 & .000 & $\begin{array}{l}10.705 \\
30.403\end{array}$ & $\begin{array}{r}106 \\
95.000\end{array}$ & $\begin{array}{l}.000 \\
.000\end{array}$ & $\begin{array}{l}12.125 \\
12.125\end{array}$ & $\begin{array}{r}1.133 \\
.399\end{array}$ & $\begin{array}{r}9.879 \\
11.333\end{array}$ & $\begin{array}{l}14.371 \\
12.917\end{array}$ \\
\hline RS & $\begin{array}{l}\text { Equal variances } \\
\text { assumed } \\
\text { Equal variances } \\
\text { not assumed }\end{array}$ & 27.895 & .000 & $\begin{array}{r}3.837 \\
10.897\end{array}$ & $\begin{array}{r}106 \\
95.000\end{array}$ & $\begin{array}{l}.000 \\
.000\end{array}$ & $\begin{array}{l}5.000 \\
5.000\end{array}$ & $\begin{array}{r}1.303 \\
.459\end{array}$ & $\begin{array}{l}2.416 \\
4.089\end{array}$ & $\begin{array}{l}7.584 \\
5.911\end{array}$ \\
\hline REC & $\begin{array}{l}\text { Equal variances } \\
\text { assumed } \\
\text { Equal variances } \\
\text { not assumed }\end{array}$ & 15.747 & .000 & $\begin{array}{l}3.416 \\
9.700\end{array}$ & $\begin{array}{r}106 \\
95.000\end{array}$ & $\begin{array}{l}.001 \\
.000\end{array}$ & $\begin{array}{l}3.125 \\
3.125\end{array}$ & $\begin{array}{l}.915 \\
.322\end{array}$ & $\begin{array}{l}1.311 \\
2.485\end{array}$ & $\begin{array}{l}4.939 \\
3.765\end{array}$ \\
\hline $\mathrm{RE}$ & $\begin{array}{l}\text { Equal variances } \\
\text { assumed } \\
\text { Equal variances } \\
\text { not assumed }\end{array}$ & 28.289 & .000 & $\begin{array}{r}6.281 \\
17.839\end{array}$ & $\begin{array}{r}106 \\
95.000\end{array}$ & $\begin{array}{l}.000 \\
.000\end{array}$ & $\begin{array}{l}6.875 \\
6.875\end{array}$ & $\begin{array}{r}1.095 \\
.385\end{array}$ & $\begin{array}{l}4.705 \\
6.110\end{array}$ & $\begin{array}{l}9.045 \\
7.640\end{array}$ \\
\hline RO & $\begin{array}{l}\text { Equal variances } \\
\text { assumed } \\
\text { Equal variances } \\
\text { not assumed }\end{array}$ & 36.902 & .000 & $\begin{array}{r}5.875 \\
16.685\end{array}$ & $\begin{array}{r}106 \\
95.000\end{array}$ & $\begin{array}{l}.000 \\
.000\end{array}$ & $\begin{array}{l}7.250 \\
7.250\end{array}$ & $\begin{array}{r}1.234 \\
.435\end{array}$ & $\begin{array}{l}4.803 \\
6.387\end{array}$ & $\begin{array}{l}9.697 \\
8.113\end{array}$ \\
\hline RI & $\begin{array}{l}\text { Equal variances } \\
\text { assumed } \\
\text { Equal variances } \\
\text { not assumed }\end{array}$ & 35.333 & .000 & $\begin{array}{r}8.916 \\
25.323\end{array}$ & $\begin{array}{r}106 \\
95.000\end{array}$ & $\begin{array}{l}.000 \\
.000\end{array}$ & $\begin{array}{l}9.000 \\
9.000\end{array}$ & $\begin{array}{r}1.009 \\
.355\end{array}$ & $\begin{array}{l}6.999 \\
8.294\end{array}$ & $\begin{array}{r}11.001 \\
9.706\end{array}$ \\
\hline $\mathrm{PI}$ & $\begin{array}{l}\text { Equal variances } \\
\text { assumed } \\
\text { Equal variances } \\
\text { not assumed }\end{array}$ & 24.231 & .000 & $\begin{array}{l}1.683 \\
4.779\end{array}$ & $\begin{array}{r}106 \\
95.000\end{array}$ & $\begin{array}{l}.095 \\
.000\end{array}$ & $\begin{array}{l}1.625 \\
1.625\end{array}$ & $\begin{array}{l}.966 \\
.340\end{array}$ & $\begin{array}{r}-.290 \\
.950\end{array}$ & $\begin{array}{l}3.540 \\
2.300\end{array}$ \\
\hline SRD & $\begin{array}{l}\text { Equal variances } \\
\text { assumed } \\
\text { Equal variances } \\
\text { not assumed }\end{array}$ & 41.222 & .000 & $\begin{array}{l}13.404 \\
38.067\end{array}$ & $\begin{array}{r}106 \\
95.000\end{array}$ & $\begin{array}{l}.000 \\
.000\end{array}$ & $\begin{array}{l}7.750 \\
7.750\end{array}$ & $\begin{array}{l}.578 \\
.204\end{array}$ & $\begin{array}{l}6.604 \\
7.346\end{array}$ & $\begin{array}{l}8.896 \\
8.154\end{array}$ \\
\hline $\mathrm{RA}$ & $\begin{array}{l}\text { Equal variances } \\
\text { assumed } \\
\text { Equal variances } \\
\text { not assumed }\end{array}$ & 36.642 & .000 & $\begin{array}{l}2.772 \\
7.873\end{array}$ & $\begin{array}{r}106 \\
95.000\end{array}$ & $\begin{array}{l}.007 \\
.000\end{array}$ & $\begin{array}{l}3.250 \\
3.250\end{array}$ & $\begin{array}{r}1.172 \\
.413\end{array}$ & $\begin{array}{r}.926 \\
2.431\end{array}$ & $\begin{array}{l}5.574 \\
4.069\end{array}$ \\
\hline Rin & $\begin{array}{l}\text { Equal variances } \\
\text { assumed } \\
\text { Equal variances } \\
\text { not assumed }\end{array}$ & 132.696 & .000 & $\begin{array}{l}10.626 \\
30.179\end{array}$ & $\begin{array}{r}106 \\
95.000\end{array}$ & $\begin{array}{l}.000 \\
.000\end{array}$ & $\begin{array}{l}10.500 \\
10.500\end{array}$ & $\begin{array}{l}.988 \\
.348\end{array}$ & $\begin{array}{l}8.541 \\
9.809\end{array}$ & $\begin{array}{l}12.459 \\
11.191\end{array}$ \\
\hline Stress & $\begin{array}{l}\text { Equal variances } \\
\text { assumed } \\
\text { Equal variances } \\
\text { not assumed }\end{array}$ & 41.551 & .000 & $\begin{array}{r}8.307 \\
23.593\end{array}$ & $\begin{array}{r}106 \\
95.000\end{array}$ & $\begin{array}{l}.000 \\
.000\end{array}$ & $\begin{array}{l}66.500 \\
66.500\end{array}$ & $\begin{array}{l}8.005 \\
2.819\end{array}$ & $\begin{array}{l}50.629 \\
60.904\end{array}$ & $\begin{array}{l}82.371 \\
72.096\end{array}$ \\
\hline
\end{tabular}




\section{Macrothink}

Table 5. Descriptive statistics of stress on basis of work experience

Group Statistics

\begin{tabular}{|ll|r|r|r|r|}
\hline & $\begin{array}{l}\text { Experience }(\mathrm{m} \\
\text { orethan5=1) }\end{array}$ & $\mathrm{N}$ & Mean & Std. Deviation & $\begin{array}{c}\text { Std. Error } \\
\text { Mean }\end{array}$ \\
\hline IRD & 0 & 24 & 4.00 & 1.022 & .209 \\
& 1 & 84 & 9.43 & 5.427 & .592 \\
\hline RS & 0 & 24 & 8.00 & 4.086 & .834 \\
& 1 & 84 & 7.43 & 4.652 & .508 \\
\hline REC & 0 & 24 & 3.50 & 2.554 & .521 \\
& 1 & 84 & 7.00 & 2.845 & .310 \\
\hline RE & 0 & 24 & 6.50 & 5.618 & 1.147 \\
& 1 & 84 & 4.43 & 3.561 & .389 \\
\hline RO & 0 & 24 & 5.00 & 1.022 & .209 \\
& 1 & 84 & 8.29 & 4.978 & .543 \\
\hline RI & 0 & 24 & 4.00 & 2.043 & .417 \\
& 1 & 84 & 6.57 & 4.652 & .508 \\
\hline PI & 0 & 24 & 5.00 & 5.108 & 1.043 \\
& 1 & 84 & 3.14 & 2.245 & .245 \\
\hline SRD & 0 & 24 & 5.50 & 1.532 & .313 \\
& 1 & 84 & 8.86 & 3.015 & .329 \\
\hline RA & 0 & 24 & 4.00 & 4.086 & .834 \\
& 1 & 84 & 6.71 & 3.714 & .405 \\
\hline Rin & 0 & 24 & 6.00 & 2.043 & .417 \\
& 1 & 84 & 8.14 & 5.028 & .549 \\
\hline Stress & 0 & 24 & 51.50 & 27.070 & 5.526 \\
& 1 & 84 & 70.00 & 34.077 & 3.718 \\
\hline
\end{tabular}


Table 6. Independent sample t-test of stress on basis of work experience

\begin{tabular}{|c|c|c|c|c|c|c|c|c|c|c|}
\hline \multicolumn{11}{|c|}{ Independent Samples Test } \\
\hline & & \multicolumn{2}{|c|}{$\begin{array}{c}\text { Levene's Test for } \\
\text { Equality of Variances }\end{array}$} & \multicolumn{7}{|c|}{ t-test for Equality of Means } \\
\hline & & \multirow[t]{2}{*}{. } & \multirow[b]{2}{*}{ Sig. } & \multirow[b]{2}{*}{$\mathrm{t}$} & \multirow[b]{2}{*}{ df } & \multirow[b]{2}{*}{ Sig. (2-tailed) } & \multirow{2}{*}{$\begin{array}{c}\text { Mean } \\
\text { Difference }\end{array}$} & \multirow{2}{*}{$\begin{array}{l}\text { Std. Error } \\
\text { Difference }\end{array}$} & \multicolumn{2}{|c|}{$\begin{array}{l}\text { 95\% Confidence } \\
\text { Interval of the } \\
\text { Difference }\end{array}$} \\
\hline & & & & & & & & & Lower & Upper \\
\hline $\mathrm{IRD}$ & $\begin{array}{l}\text { Equal variances } \\
\text { assumed } \\
\text { Equal variances } \\
\text { not assumed }\end{array}$ & 26.634 & .000 & $\begin{array}{l}-4.860 \\
-8.647\end{array}$ & $\begin{array}{r}106 \\
99.348\end{array}$ & $\begin{array}{l}.000 \\
.000\end{array}$ & $\begin{array}{l}-5.429 \\
-5.429\end{array}$ & $\begin{array}{r}1.117 \\
.628\end{array}$ & $\begin{array}{l}-7.643 \\
-6.674\end{array}$ & $\begin{array}{l}-3.214 \\
-4.183\end{array}$ \\
\hline RS & $\begin{array}{l}\text { Equal variances } \\
\text { assumed } \\
\text { Equal variances } \\
\text { not assumed }\end{array}$ & .026 & .872 & $\begin{array}{l}.544 \\
.585\end{array}$ & $\begin{array}{r}106 \\
41.613\end{array}$ & $\begin{array}{l}.587 \\
.562\end{array}$ & $\begin{array}{l}.571 \\
.571\end{array}$ & $\begin{array}{r}1.050 \\
.976\end{array}$ & $\begin{array}{l}-1.510 \\
-1.400\end{array}$ & $\begin{array}{l}2.653 \\
2.542\end{array}$ \\
\hline REC & $\begin{array}{l}\text { Equal variances } \\
\text { assumed } \\
\text { Equal variances } \\
\text { not assumed }\end{array}$ & .087 & .769 & $\begin{array}{l}-5.430 \\
-5.769\end{array}$ & $\begin{array}{r}106 \\
40.788\end{array}$ & $\begin{array}{l}.000 \\
.000\end{array}$ & $\begin{array}{l}-3.500 \\
-3.500\end{array}$ & $\begin{array}{l}.645 \\
.607\end{array}$ & $\begin{array}{l}-4.778 \\
-4.726\end{array}$ & $\begin{array}{l}-2.222 \\
-2.274\end{array}$ \\
\hline RE & $\begin{array}{l}\text { Equal variances } \\
\text { assumed } \\
\text { Equal variances } \\
\text { not assumed }\end{array}$ & 44.342 & .000 & $\begin{array}{l}2.185 \\
1.711\end{array}$ & $\begin{array}{r}106 \\
28.479\end{array}$ & $\begin{array}{l}.031 \\
.098\end{array}$ & $\begin{array}{l}2.071 \\
2.071\end{array}$ & $\begin{array}{r}.948 \\
1.211\end{array}$ & $\begin{array}{r}.192 \\
-.407 \\
\end{array}$ & $\begin{array}{l}3.951 \\
4.550\end{array}$ \\
\hline $\mathrm{RO}$ & $\begin{array}{l}\text { Equal variances } \\
\text { assumed } \\
\text { Equal variances } \\
\text { not assumed }\end{array}$ & 74.085 & .000 & $\begin{array}{l}-3.204 \\
-5.647\end{array}$ & $\begin{array}{r}106 \\
101.324\end{array}$ & $\begin{array}{l}.002 \\
.000\end{array}$ & $\begin{array}{l}-3.286 \\
-3.286\end{array}$ & $\begin{array}{r}1.026 \\
.582\end{array}$ & $\begin{array}{l}-5.319 \\
-4.440\end{array}$ & $\begin{array}{l}-1.252 \\
-2.132\end{array}$ \\
\hline $\mathrm{RI}$ & $\begin{array}{l}\text { Equal variances } \\
\text { assumed } \\
\text { Equal variances } \\
\text { not assumed }\end{array}$ & 14.366 & .000 & $\begin{array}{l}-2.629 \\
-3.914\end{array}$ & $\begin{array}{r}106 \\
88.072\end{array}$ & $\begin{array}{l}.010 \\
.000\end{array}$ & $\begin{array}{l}-2.571 \\
-2.571\end{array}$ & $\begin{array}{l}.978 \\
.657\end{array}$ & $\begin{array}{l}-4.510 \\
-3.877\end{array}$ & $\begin{array}{r}-.632 \\
-1.266\end{array}$ \\
\hline $\mathrm{PI}$ & $\begin{array}{l}\text { Equal variances } \\
\text { assumed } \\
\text { Equal variances } \\
\text { not assumed }\end{array}$ & 157.907 & .000 & $\begin{array}{l}2.589 \\
1.734\end{array}$ & $\begin{array}{r}106 \\
25.587\end{array}$ & $\begin{array}{l}.011 \\
.095\end{array}$ & $\begin{array}{l}1.857 \\
1.857\end{array}$ & $\begin{array}{r}.717 \\
1.071\end{array}$ & $\begin{array}{r}.435 \\
-.346 \\
\end{array}$ & $\begin{array}{l}3.279 \\
4.060\end{array}$ \\
\hline SRD & $\begin{array}{l}\text { Equal variances } \\
\text { assumed } \\
\text { Equal variances } \\
\text { not assumed }\end{array}$ & 2.410 & .124 & $\begin{array}{l}-5.253 \\
-7.396 \\
\end{array}$ & $\begin{array}{r}106 \\
76.183\end{array}$ & $\begin{array}{l}.000 \\
.000\end{array}$ & $\begin{array}{l}-3.357 \\
-3.357\end{array}$ & $\begin{array}{l}.639 \\
.454\end{array}$ & $\begin{array}{l}-4.624 \\
-4.261\end{array}$ & $\begin{array}{l}-2.090 \\
-2.453\end{array}$ \\
\hline RA & $\begin{array}{l}\text { Equal variances } \\
\text { assumed } \\
\text { Equal variances } \\
\text { not assumed }\end{array}$ & 4.489 & .036 & $\begin{array}{l}-3.088 \\
-2.927\end{array}$ & $\begin{array}{r}106 \\
34.609\end{array}$ & $\begin{array}{l}.003 \\
.006\end{array}$ & $\begin{array}{l}-2.714 \\
-2.714\end{array}$ & $\begin{array}{l}.879 \\
.927\end{array}$ & $\begin{array}{l}-4.457 \\
-4.598\end{array}$ & $\begin{array}{l}-.971 \\
-.831\end{array}$ \\
\hline Rin & $\begin{array}{l}\text { Equal variances } \\
\text { assumed } \\
\text { Equal variances } \\
\text { not assumed } \\
\end{array}$ & 24.624 & .000 & $\begin{array}{l}-2.035 \\
-3.110\end{array}$ & $\begin{array}{r}106 \\
93.713\end{array}$ & $\begin{array}{l}.044 \\
.002\end{array}$ & $\begin{array}{l}-2.143 \\
-2.143\end{array}$ & $\begin{array}{r}1.053 \\
.689\end{array}$ & $\begin{array}{l}-4.231 \\
-3.511\end{array}$ & $\begin{array}{l}-.055 \\
-.775\end{array}$ \\
\hline Stress & $\begin{array}{l}\text { Equal variances } \\
\text { assumed } \\
\text { Equal variances } \\
\text { not assumed }\end{array}$ & .146 & .703 & $\begin{array}{l}-2.445 \\
-2.778\end{array}$ & $\begin{array}{r}106 \\
45.933\end{array}$ & $\begin{array}{l}.016 \\
.008\end{array}$ & $\begin{array}{l}-18.500 \\
-18.500\end{array}$ & $\begin{array}{l}7.565 \\
6.660\end{array}$ & $\begin{array}{l}-33.498 \\
-31.907\end{array}$ & $\begin{array}{l}-3.502 \\
-5.093\end{array}$ \\
\hline
\end{tabular}

\section{Discussion}

The stress level of faculty members is increasing. They experience high role stress as shown in Table 7. The major area of concerns is Inter role distance, Self role distance and Resource Inadequacy. This is probably because of the changing expectation of different stakeholders from the faculty members of higher educational institutions. The research shows that the faculty with more than 5 years of experience in this field exhibits higher stress, this seems plausible, as it has been observed that there is lot of pressure to perform, not only in teaching but also in research, for the career progression. 
Table 7. Descriptive statistics of role stress

\begin{tabular}{|l|r|r|r|r|r|}
\hline \multicolumn{7}{|c|}{ Descriptive Statistics } \\
\hline IRD & N & Minimum & Maximum & Mean & Std. Deviation \\
RS & 108 & 2 & 19 & 8.22 & 5.311 \\
REC & 108 & 0 & 13 & 7.56 & 4.521 \\
RE & 108 & 1 & 12 & 6.22 & 3.134 \\
RO & 108 & 1 & 12 & 4.89 & 4.168 \\
RI & 108 & 0 & 14 & 7.56 & 4.619 \\
PI & 108 & 0 & 14 & 6.00 & 4.341 \\
SRD & 108 & 0 & 10 & 3.56 & 3.181 \\
RA & 108 & 4 & 15 & 8.11 & 3.085 \\
Rin & 108 & 0 & 12 & 6.11 & 3.947 \\
Stress & 108 & 2 & 17 & 7.67 & 4.616 \\
Valid N (listwise) & 108 & 19 & 125 & 65.89 & 33.437 \\
\hline
\end{tabular}

\section{Conclusion}

The field of education is not unscathed of VUCA world and hence the high role stress of the faculty members is observed. With the increasing expectations, the adequate resource supply should be maintained and the congruence in other role requirements should be given due importance.

\section{References}

Areekkuzhiyil, S. (2011). Organisational Stress among Faculty Members of Higher Education Sector.

Arnold, J. H., \& Feldman, C. (1986). Organizational behaviour. New York: McGraw Hill.

Chaudhry, A. Q. (2013). Analysis of Occupational Stress of University Faculty to Improve the Quality of Their Work. Journal of Quality and Technology Management, IX(I), 12-29.

Cooper, C., Dewe, P. J., \& O'Driscoll, M. P. (2001). Organizational stress: A review and critique of theory, research, and applications. Thousand Oaks, CA: Sage.

Dinham, S., \& Scott, C. (1998). An international comparative study of teacher satisfaction, motivation and health: Australia, England, and New Zealand. American Educational Research Association.

Gmelch, W. H. (1993). Coping with faculty stress. New York: Sage Publications. http://dx.doi.org/10.4135/9781483326313

Gmelch, W. H., Willse, P. K., \& Lourich, N. P. (1986). Dimensions of stress among university faculty: Factor analytic results from a national study. Research in Higher Education. http://dx.doi.org/10.1007/BF00992075 
Jreige, T. (2011). Stress and Coping Strategies of Faculty Members. HEIC.

Kavitha, P. (2012). Organisational Role Stress among College Faculties: An Empirical Study. Global Management Review, 6(4).

Kousar, S., Fatima, N., \& Bashir, F. (2004). A study of stress management strategies adopted by elementary school principles. University of the Punjab, Lahore.

Kyriacou, C. (2001). Teacher stress: Directions for future research. Educational Research, 53(1), 27-35.

Madhu, K., Ananda, T. V., \& Rao, A. N. (1990). Role stress: differential influences of some antecedent factors. Psy. Stu., 35(1), 28-35.

Manthei, R. (1988). School counselors and job related stress. New Zealand Educational Studies, 22, 189-200.

Pareek, U. (1983). Training Instruments in HRD \& OD. Tata Mc Graw Hill Publication.

Pareek, U. (2002). Training Instruments in HRD \&OD. Tata Mc Graw Hill Publication.

Pareek, U. (2004). Training Instruments in HRD \&OD. Tata Mc Graw Hill Publication.

Pareek, U., \& Purohit, S. (2010). Training Instruments in HRD \&OD (3rd ed.). Tata Mc Graw Hill Publication.

Ravichandran, R., \& Rajendran, R. (2007). Perceived Sources of Stress among the Teachers. Journal of the Indian Academy of Applied Psychology, 33(1), 133-136.

Repetti, R. L., \& Wood, J. (1997). The effect of daily stress at work on mothers' interaction with preschoolers. Journal of family psychology, 11, 90-108. http://dx.doi.org/10.1037/0893-3200.11.1.90

Slišković, A, \& Maslić, S. D. (2011). Work Stress among University Teachers. Arh Hig Rada Toksikol, 62, 299-307. http://dx.doi.org/10.2478/10004-1254-62-2011-2135

Spielberger, C. D., \& Reheiser, E. C. (1994). The job stress survey: Measuring gender differences in occupational stress. Journal of Social Behavior and Personality, 9, 199-218.

Thelma Smith Patterson Sanders. (1989). The perceptions of stress among faculty members in higher education at six selected Tennessee State Board of Regents universities. ETD Collection for Tennessee State University, Paper AAI9017229.

\section{Copyright Disclaimer}

Copyright for this article is retained by the author(s), with first publication rights granted to the journal.

This is an open-access article distributed under the terms and conditions of the Creative Commons Attribution license (http://creativecommons.org/licenses/by/3.0/). 